agents, anti-Alzheimer drugs, and insecticides. A better understanding of its molecular mode of action thus goes beyond academic interest, and may be beneficial to the design of anti-Alzheimer drugs, antidotes against OP poisoning and novel insecticides. We have solved crystal structures of conjugates with Torpedo californica AChE (TcAChE) of the potent nerve agent, soman. Soman reacts covalently with the active-site serine to form an initial conjugate (the 'non-aged' conjugate), that can be reactivated by suitable oximes. This conjugate subsequently undergoes a dealkylation reaction known as 'aging', that produce a conjugate that can no longer be reactivated (the 'aged conjugate') [1]. X-ray crystallography data collection performed at the ESRF (Grenoble, France), permitted solution of the structure of both conjugates. Since 'aging' reaction is fast at room temperature, the crystal-soaking time prior to cryo-cooling had to be reduced to minutes in order to trap the "nonaged' conjugate. The structures obtained supply a structural basis for rational designing of more oxime reactivators that may serve as antidotes of OP intoxication.

[1] Millard, C.B. et al., "Crystal structures of aged phosphonylated acetylcholinesterase: nerve agent reaction products at the atomic level" Biochemistry 38, $7032-7039$ (1999)

\section{MS03 P13}

Crystallographic study of E. coli WrbA protein in complex with its flavin cofactor Julie Wolfova, ${ }^{\mathrm{a}, \mathrm{b}}$, Jiri Brynda ${ }^{\mathrm{a}, \mathrm{c}}$, Jannette Carey ${ }^{\mathrm{d}}$, Ivana Kuta Smatanova ${ }^{\mathrm{a}, \mathrm{b}}$. Institute of Physical Biology, University of South Bohemia Ceske Budejovice, Nove

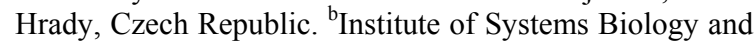
Ecology, Academy of Science of the Czech Republic,

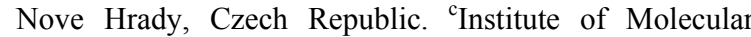
Genetics, Academy of Sciences of the Czech Republic, Prague, Czech Republic. ${ }^{\mathrm{d}}$ Chemistry Department, Princeton University, Princeton, USA.

E-mail: julinka.w@tiscali.cz

\section{Keywords: crystallography of biological macromolecules, flavoproteins, protein-ligand complexes}

The currently studied protein WrbA from Escherichia coli belongs to a new family of multimeric flavoproteins [1,2], that contain flavin mononucleotide (FMN) as a physiological cofactor. The latest observations revealed that E. coli WrbA and its homologues exhibit the $\mathrm{NAD}(\mathrm{P}) \mathrm{H}$ :quinone oxidoreductase activity, which is proposed to be implicated in protection mechanisms against oxidative stress [3]. Crystallographic study of the $E$. coli $\mathrm{WrbA}$ is aimed at proper characterization of the new protein family with respect to its recently revealed oxidoreductase activity. Recombinant WrbA protein was expressed in E. coli CY15071(1DE3) cells and purified as described previously [2]. Pure WrbA protein was obtained without bound cofactor, because of the loss of FMN during purification. The complex of WrbA protein with the FMN cofactor for crystallization was prepared by incubation of pure WrbA with equimolar concentration of FMN. Crystallization was carried out at $285 \mathrm{~K}$ by the sitting-drop vapor-diffusion technique, with droplets containing equal parts of protein and precipitant solution. Yellow, well-formed tetragonal crystals of WrbA protein in complex with its flavin cofactor were obtained from several crystallization conditions. Yielding of diffraction- quality crystals without additional optimzation steps shows the positive influence of FMN cofactor on crystallization of WrbA protein. This effect reflects FMN as a potential stabilizer of the protein structure. Diffraction data for the crystals reported here were collected at $100 \mathrm{~K}$ at synchrotron DESY, beamline X13 (EMBL) with an Xray wavelength of $0.805 \AA$. The different crystallization conditions yielded two forms of primitive tetragonal crystals having different space groups (P43212; P41212) and different unit-cell dimensions $(\mathrm{a}=\mathrm{b}=94.351 \AA$, $\mathrm{c}=$

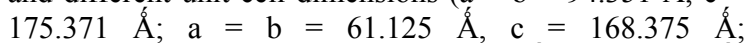
respectively). The resolutions of $2.60 \AA$ and $1.99 \AA$, respectively, were reached. The diffraction limit of the better-diffracting crystal represents the best resolution reported to date for WrbA protein in complex with FMN. Solving of the structures from both data sets is in progress.

This work is supported by the Ministry of Education of the Czech Republic (projects: Kontakt ME640, MSM6007665808, LC06010) and by the Academy of Sciences of the Czech Republic (AV0Z60870520)

[1] Grandori R., Carey J., Protein Sci., 1994, 3, 2185.

[2] Grandori R., Khalifah P., Boice J.A., Fairman R., Giovanelli

K., Carey J., J. Biol. Chem., 1998, 273, 20960.

[3] Patridge E.V., Ferry J.G., J. Bacteriol., 2006, 188, 3498.

\section{MS03 P14}

Carbohydrate-binding of the starch binding domain of Rhizopus oryzae glucoamylase in complex with $\beta$-cyclodextrin and maltoheptaose

Yuj-Ju Sun ${ }^{\mathrm{a}}$, Jung-Yu Tunga ${ }^{\mathrm{a}}$, Yen-Yi Liu ${ }^{\mathrm{a}}$, Wei-I Chou, Margaret D.-T. Chang ${ }^{\mathrm{b}}$, Institute of Bioinformatics and Structural Biology and ${ }^{\mathrm{b}}$ Institute of Molecular and Cellular Biology, National Tsing Hua University, Hsinchu 300, Taiwan, ROC._E-mail: yjsun@life.nthu.edu.tw

Keywords: Rhizopus oryzae glucoamylase, starch binding domain, carbohydrate binding

Glucoamylase hydrolyses starch and polysaccharides to $\beta$ D-glucose. Rhizopus oryzae glucoamylase consists of two functional domains, an N-terminal starch binding domain (SBD) and a C-terminal catalytic domain. The two domains are connected by an O-glycosylated linker. The crystal structures of the SBD in complex with a cyclic carbohydrate, $\beta$-cyclodextrine, and a linear carbohydrate, maltoheptaose, were determined. The overall structures of the SBD complexes belong to a $\beta$-sandwich fold with an immunoglobulin-like structure and two carbohydratebinding sites were observed. Site I is created by several conserved aromatic residues, Trp47, Tyr83, and Tyr94, to form a broad, flat, and firm hydrophobic surface. In addition to the hydrophobic interaction, numerous asparagines residues are involved in the hydrophilic interactions to carbohydrate. Site II is built up by Tyr32 and Phe58 to produce a protruded and narrow binding environment. Site I undergoes a bigger conformational change than site II upon the carbohydrate binding. 